\title{
Corsi di formazione accreditati ECM: Mindfulness, Psicodiagnostica, BES e DSA
}

\section{CORSO BIENNALE DI PSICODIAGNOSTICA NOVEMBRE 2016}

Prossimi alla conclusione della seconda edizione, ripartirà, nel mese di novembre 2016, la Terza Edizione del Corso Biennale di Psicodiagnostica, promosso dalla FederPsi-SCint, presso la sede di Via Ravenna 24 a Roma, rivolto a psicologi, medici, specializzati e specializzandi in psichiatria, psicoterapia, studenti o laureati in psicologia con rilascio di 50 crediti ECM. Il percorso formativo ha la finalità di contribuire all'acquisizione di competenze relative alla somministrazione e interpretazione dei più diffusi e utilizzati test psicologici in ambito clinico per l'età adulta e l'età evolutiva in funzione dei più recenti criteri nosografici secondo il nuovo DSM 5. Il corso comprende $\mathbf{1 6}$ moduli teorico-pratici in aula attraverso una didattica di tipo interattiva con simulazioni di somministrazione dei test studiati, esercitazioni in gruppo, indicazioni sulla stesura del report finale e discussione di casi clinici. Il programma didattico si articola in tre grandi tre aree: i test di personalità e i test cognitivi; il test di Rorschach; gli strumenti di valutazione in età evolutiva: test proiettivi, attaccamento e infanzia. Al termine del corso i professionisti saranno in grado di valutare la condizione di normalità e/o psicopatologia di un soggetto e descrivere tale condizione in termini di risorse (personali, interpersonali, sociali) e criticità; scegliere la batteria psicodiagnostica più adeguata a misurare abilità e caratteristiche di personalità in rapporto al tipo di domanda (psicologia clinica, psicologia giuridica, della famiglia, dello sviluppo) e stendere una relazione psicodiagnostica adeguata ed esaustiva. Il Comitato scientifico è costituito da un corpo qualificato di professionisti: prof. Tonino Cantelmi e collaboratori, prof.ssa Maria Beatrice Toro e collaboratori, prof.ssa Michela 
Pensavalli, dott. Filippo De Santis, dott. Marco Scicchitano, dott. Emiliano Lambiase, dott.ssa Marina D'Angeli, dott. Antonio Minopoli, prof.ssa Barbara Turella.

\section{CORSO ANNUALE DI FORMAZIONE ALLA MindFULNESS FEBBRAIO 2016}

Nel mese di febbraio 2016 è partito il primo corso di formazione per istruttori di mindfulness elaborato secondo le linee guida dell'UK Network for mindfulness, in collaborazione con il Secondo Centro di Terapia Cognitivo Interpersonale, sito in Roma, via Nomentana 257. Tale corso nasce dalla esigenza di approfondire una disciplina che si configura come uno strumento valido d'integrazione alla psicoterapia, ai diversi contesti clinici ed educativi aprendo nuovi orizzonti per la cura del benessere psicofisico della persona. La proposta formativa si declina in $\mathbf{8}$ moduli dal carattere soprattutto esperienziale a cui si integrano le cornici teoriche di riferimento al fine di offrire ai partecipanti una formazione adeguata che dia loro le basi per la conduzione di protocolli educativi di Mindfulness Based Stress Reduction (MBSR). Tale formazione è rivolta alla categoria professionale di medici e psicologi, laureati e laureandi, praticanti e non di mindfulness. Al termine del corso, nel mese di settembre 2016, è previsto un esame finale con il rilascio di un diploma che attesta la qualifica di "Istruttore di protocolli Mindfulness". Il Comitato scientifico si compone di un insegnante guida, dott. Stefano Serafinelli e di corpo qualificato di docenti: prof. Tonino Cantelmi, prof.ssa Maria Beatrice Toro, dott.ssa Roberta Necci, prof.ssa Paola De Bartolo, prof. Debora Cutuli.

\section{INDIVIDUAZIONE E GESTIONE DEI BES - BISOGNI EDUCATIVI SPECIALI -EDSA}

2017

I bisogni educativi rappresentano un costrutto attuale, che affonda le sue radici nell'ambito della teoria dei bisogni, nonché nel contesto cognitivo comportamentale della psicologia dell'apprendimento. La scuola di oggi è chiamata, sempre più, a rispondere a una varietà di bisogni dei propri alunni talvolta manifestati in modo più evidente, in altri casi in modo più celato e sfumato ma non per questo meno significativo. Infatti, 
accanto alle forme più evidenti di disabilità c'è un crescente numero di bambini che nonostante non abbia una certificazione, presenta dei Bisogni Educativi Speciali (disturbi specifici dell'apprendimento, difficoltà psicologiche, comportamentali e affettivo-relazionale, di svantaggio sociale, culturale e linguistico, gli alunni stranieri, ecc.), destando le preoccupazioni di insegnanti e genitori. E sono proprio gli insegnanti a dover affrontare la complessità e le difficoltà dell'eterogeneità del contesto classe, che si osserva nei processi di apprendimento, negli stili di pensiero, relazionali, sociali, culturali. Ė quindi necessaria una formazione continua per gli insegnanti che frequentemente sono i primi educatori a cogliere una difficoltà negli alunni e comunicarla alle famiglie per una presa in carico quanto più tempestiva possibile. Per loro è necessario possedere strumenti utili a saper cogliere in tempo le difficoltà in classe e attivare tutte le risorse possibili per impostare interventi psicoeducativi e didattici più funzionali in un'ottica collaborativa tra il clinico o il servizio che ha in carico l'alunno e la scuola.

È da tali analisi ed esigenza che la FederPsi ha promosso con grande entusiasmo e come una sfida la prima edizione del corso di formazione con rilascio di ECM: "Individuazione precoce e gestione dei BES - Bisogni Educativi Speciali - e DSA" in collaborazione con l'ARPA - Associazione Italiana ricerca Psicosi e Autismo - Ente accreditato dal MIUR per la professione degli insegnanti, con l'obiettivo di formare, fare incontrare e dialogare le diverse figure professionali che ruotano attorno al bambino con BES: i clinici (neuropsichiatri infantili, psicologi, psicoterapeuti, logopedisti ecc.) che si occupano di individuazione/diagnosi e trattamento e gli insegnanti che sono chiamati a orientare le loro strategie di intervento educative e didattiche all'interno di una cornice relazionale di qualità tra le varie figure coinvolte. Alla conclusione del corso verrà rilasciato un attestato riconosciuto per entrambe le categorie professionali in qualità di "Esperto nell'individuazione e gestione dei BES e DSA".

Comitato scientifico: prof. Tonino Cantelmi, prof.ssa Maria Beatrice Toro, dott. Filippo Bianchini, dott.ssa Mara Bruno, dott.ssa Loredana Canzano, dott.ssa Valeria Carpino, dott.ssa Bianca Crocamo, dott.ssa Debora Cutuli, prof.ssa Paola De Bartolo, dott.ssa Michela De Luca, dott.ssa Amelia Palladino.

È dopo il successo della prima edizione conclusa a giugno 2016, che ha visto una numerosa partecipazione e interesse di psicologi, psicoterapeuti, insegnanti, insegnanti di sostegno e pedagogisti, che con altrettanto entusiasmo saremo pronti a ripartire nell'anno 2017 con una seconda edizione.

www.cognitivo-interpersonale.it 